\title{
Comparison Between the Use of a Genexpert Device and Conventional Diagnostic Methods in the Detection of Mycobacterial Tuberculosis and Its Resistance to Drugs
}

\author{
Ahmed E. Elsayed ${ }^{(1)}$, Mostafa Y. El-Mishad ${ }^{(1)}$, Mahmoud A. Mohamed ${ }^{(1)}$, \\ Ibrahim M. El-hosiny (1),Ahmed Abdel Tawab ${ }^{(1)}$, Ghanem A. Mohamed ${ }^{(2)}$ \\ ${ }^{(1)}$ Department of Medical Microbiology and Immunology and ${ }^{(2)}$ Department of chest diseases, Faculty of \\ Medicine, Al-Azhar University, Cairo, Egypt \\ Corresponding author: Ahmed E. Elsayed; Mobile: 01003967843; Email: elamgad@ hotmail.com,
} Orchid id: 0000-0002-9721-3584

\begin{abstract}
Background: Mycobacterium tuberculosis (MTB) is the causative agent of tuberculosis (TB), which remains the leading cause of morbidity and mortality worldwide. The emergence of drug-resistant strains of MTB has put status of TB to threatening levels. Aim of the Work: was to detect MTB along with rifampicin (RIF) resistance using Genexpert (MTB/RIF). Its diagnostic, sensitivity and specificity were evaluated by comparing with conventional technique. Patients, Materials and Methods: This prospective study was conducted on two hundred and seven Egyptian patients at Abbasia Chest Diseases Hospital, from November 2016 to December 2017, and comprised clinically and radiologically diagnosed TB suspected cases. This study was approved by the Ethical Committee of faculty of medicine, Alazhar University and the Ethical Committee of Ministry of Health and after Verbal consents from the patients or their parents were taken. Pulmonary specimens (sputum and bronchial lavage) and pleural effusion as an extra-pulmonary specimen were included. All samples collected were sent to TB laboratory of Abbasia Chest Diseases Hospital for further analysis. Result: Out of the 26 Genexpert (MTB/RIF) positive samples for MTBC, 3 (11.54\%) showed RIF resistance and diagnosed as MDR-TB. Using LJ and MGIT cultures for drug sensitivity test (DST) on 31 and 34 positive TB samples; respectively, the same three specimens showed resistance to rifampicin (RIF). Four positive specimens were also resistant to streptomycin (STR) using the previously mentioned cultures. Moreover, resistant to INH was reported in five positive TB samples using the same cultures. Finally, it was found that all positive specimens were sensitive to Ethambutol (ETH). Conclusion: Although the conventional methods remain the gold standard for diagnosing pulmonary $\mathrm{TB}$, delayed diagnostic times demand for more rapid and sensitive nucleic acid amplification techniques. Genexpert (MTB/RIF) assay is simple, rapid and accurate method for detecting mycobacterial tuberculosis.
\end{abstract}

Keywords: genexpert device, mycobacterial tuberculosis, MDR.

\section{INTRODUCTION}

The mycobacteria are rod-shaped, aerobic bacteria that do not form spores. Although they do not stain readily, once stained they resist decolorization by acid or alcohol and therefore called "acid-fast" bacilli. Mycobacterium tuberculosis causes tuberculosis and is a very important pathogen of humans. Mycobacterium leprae causes leprosy. Mycobacterium aviumintracellulare (M. avium complex, or MAC) and other non-tuberculous mycobacteria frequently infect patients with acquired immunodeficiency syndrome "AIDS" are opportunistic pathogens in other immuno-compromised persons, and occasionally cause disease in patients with normal immune systems. There are more than 125 Mycobacterium species ${ }^{(\mathbf{1})}$.

Mycobacterium Tuberculosis in tissue, tubercle bacilli are thin straight rods measuring about $0.4 \times 3 \mu \mathrm{m}$. On artificial media, coccid and filamentous forms are seen with variable morphology from one species to another. Mycobacteria cannot be classified as either grampositive or gram-negative. Once stained by basic dyes they cannot be decolorized by alcohol, regardless of treatment with iodine. True tubercle bacilli are characterized by "acid-fastness" $95 \%$ ethyl alcohol containing 3\% hydrochloric acid (acid-alcohol) quickly decolorizes all bacteria except the mycobacteria. Acid-fastness depends on the integrity of the waxy envelope ${ }^{(\mathbf{1})}$.

The term tuberculosis (TB) broad ranges of clinical illness caused by Mycobacterium tuberculosis or less commonly Mycobacterium bovis and other types of mycobacteria (2). Mycobacterium tuberculosis which usually attacks the lungs, it can attack any part of the body such as the kidney, spine, and brain. Not everyone infected with tubercle bacilli becomes sick. That explains the presence of two TB-related conditions: latent TB infection (LTBI) and TB disease. If not treated properly, TB disease can be fatal ${ }^{(3)}$.

According to World Health Organization $^{(4)}$ there were 7974 newly discovered tuberculosis cases in Egypt in 2016 (4545 pulmonary cases and 3429 are extra pulmonary), 174 of them confirmed as multidrug resistant organisms by the conventional culture method. 
In high-incidence countries, pulmonary TB control relies on passive case finding among individuals self-presenting to health care facilities, followed by either diagnosis based on clinical symptoms or laboratory diagnosis using sputum smear microscopy. Serial sputum specimens are required (one taken on the spot and the following specimen sent in next days), which means that the people are asked to make repeated visits to the health care center for specimen delivery and collection of results. For many patients, the costs of repeated visits to health care facilities are prohibitive, and patient dropout is a significant problem. In addition, the sensitivity of sputum smear microscopy has been reported to vary (range, 20 to $80 \%$ ), often depending on the diligence with which specimens are collected, smears are made, and stained smears are examined ${ }^{(5)}$.

Multidrug-resistant tuberculosis (MDRTB) is defined as resistance to both isoniazid (INH) and rifampicin (RIF), and extensively drugresistant tuberculosis (XDR-TB) is defined as MDR-TB with additional resistance to any fluoroquinolone and to at least one of three injectable drugs used for TB treatment: capreomycin, kanamycin, or amikacin ${ }^{(6)}$.

The Xpert MTB/RIF assay is a fully automated molecular diagnostic test for TB disease developed to detect Mycobacterium tuberculosis complex (MTBC) DNA and mutations associated with rifampicin (RIF) resistance (a reliable proxy for MDR-TB) directly from sputum and other specimens in less than 2 hours, and it minimizes staff manipulation and biosafety risk, Moreover, its ability to detect smear-negative TB provides a significant advantage, and significantly improves the likelihood of timely treatment initiation. Conventional culture and drug-susceptibility testing [DST] are still required to complete the multi-drug resistance profile to the remaining antituberculosis drugs and to monitor the treatment provided ${ }^{(7)}$. The Xpert MTB/RIF test used with the Cepheid Genexpert巴 System is a semi-quantitative nested real-time polymerase chain reaction (PCR) in-vitro diagnostic test for: 1) the detection of Mycobacterium tuberculosis complex DNA in samples or concentrated sediments, that are either acid-fast bacilli (AFB) smear positive or negative; and 2) the detection of rifampicin resistance associated mutations of the rpoB gene in samples from patients at risk for rifampicin resistance.

The aim of this work was to throw a light on the importance of Genexpert assay as a rapid ad reliable test for the diagnosis of Mycobacterium tuberculosis infection in Egyptian patients. Also, the comparison between this technique and the conventional diagnostic methods was evaluated.
Moreover the sensitivity and the specificity Genexpert (MTB/RIF) technique was estimated comparing to conventional diagnostic procedures

\section{PATIENTS, MATERIALS AND METHODS}

This prospective study included a total of 207 Egyptian patients (117 males and 90 females) with clinically and radiologically suspected pulmonary TB, attending at Outpatient Clinics of Abbasia Chest Diseases Hospital. Approval of the Ethical Committee of Faculty of Medicine, Alazhar University and the Ethical Committee of Ministry of Health and a written informed consent from all the subjects or their parents were obtained. This study was conducted between November 2016 and December 2017.

The collected specimens (sputum, inducted sputum and bronchial lavage and pleural effusion) were processed at the Department of Microbiology Laboratory, Abbasia Chest Diseases Hospital. These samples were examined by: ZiehlNeelsen stain, Lowenstein-Jensen culture, Mycobacterial growth indicator tube (MGIT) culture and Genexpert to detect mycobacterial nucleic acid and sensitivity to rifampicin.

Any growth on culture, the identification was done to ensure that it is Mycobacterium tuberculosis complex using BECTON DICKINSON (B.D.) Identification Card and morphology of the colony. The sensitivity to rifampicin on MGIT was also done. All techniques were compared including time and the accuracy of the results. For resistant strains to rifampicin by GeneXpert, sensitivity to INH, streptomycin, and ethambutol were done on MGIT ${ }^{(\mathbf{8})}$.

\section{Gene Xpert:}

\section{MATERIALS}

GeneXpert System equipped with GX2.1 software/computer/printer/barcode (Cepheid Inc., Sunnyvale, USA).GeneXpert Cartridge Single-use disposable Xpert MTB/RIF cartridges, Sample reagent (provided in Xpert MTB/RIF kit), $8 \mathrm{ml}$ volume pack per each cartridge, permanent marker. Sterile disposable transfer pipettes with single mark for minimum volume of sample transfer to cartridge (provided in Xpert MTB/RIF kit), sterile screw-capped specimen collection containers contain decontaminated specimen and disinfectant at sufficient concentration (diluted chlorine 1:5).Sterile pipettes (Pasteur)

\section{METHODS}

One milliliter from Decontaminated specimen was added to 3 milliliter from sample reagent. The mixture was vortexes for 20 times then was left to rest for ten minutes. The mixture 
was vortexes again for 20 times; then was left to rest for five minutes. Two milliliters from mixture were put in GeneXpert Cartridge by sterile Pasteur. The accession number was written by permanent marker. Barcode was scanned by machine. The cartridge was entered to machine; then closed the door. When test was ending the door was opened. The cartridge was removed from machine. The report was printed and classify specimen to positive and negative and if it is resistant to rifampicin or not. Sometimes when bacterial load was very low cannot identify if rifampicin was sensitive or resistant ${ }^{(\mathbf{9})}$.

\section{Statistical analysis}

Recorded data were analyzed using the statistical package for social sciences, version 20 (SPSS Inc., Chicago, Illinois, USA). Quantitative data were expressed as mean \pm standard deviation (SD). Qualitative data were expressed as frequency and percentage.

The following tests were done:

A one-way analysis of variance (ANOVA) when comparing between more than two means, Chi-square $\left(\mathrm{X}^{2}\right)$ test of significance was used in order to compare proportions between two qualitative parameters.

Evaluation of Diagnostic Performance:

- Sensitivity $=($ true $+v e) /[($ true $+v e)+($ false $-\mathrm{ve})]$.

- $\quad$ Specificity $=($ true $-v e) /[($ true $-v e)+$ (false +ve)].

- $\quad$ PPV (Positive Predictive value $)=$ (true $+v e) /[($ true $+v e)+($ false $+v e)]$.

- $\quad$ NPV (Negative Predictive value $)=$ (true $-\mathrm{ve}) /[($ true $-\mathrm{ve})+($ false $-\mathrm{ve})]$.

- AUC (Area Under the Curve): the ratio of the true positive and true negative on all patient

- The confidence interval was set to $95 \%$ and the margin of error accepted was set to 5\%. So, the p-value was considered significant as the following:

- Probability (P-value)

- P-value $<0.05$ was considered significant.

- P-value <0.001 was considered as highly significant.

- P-value >0.05 was considered insignificant ${ }^{(4)}$.

This study group included 117 male (56.5\%) and 90 female (43.5\%) of gender, with increase percent of suspected male patients than female patients, also their age ranged age $4-90$ with mean age $36.72 \pm 20.79$

Table (1): Gender and age distribution of the study group.

\begin{tabular}{|l|l|}
\hline Demographic Data & Total $(\mathbf{N}=\mathbf{2 0 7})$ \\
\hline Gender & \\
Male & $117(56.5 \%)$ \\
Female & $90(43.5 \%)$ \\
\hline Age (years) & \\
Range & $4-90$ \\
Mean \pm SD & $36.72 \pm 20.79$ \\
\hline
\end{tabular}

New patients' group type included most cases in the study (93.2\%), this may refer to an increasing reemergence of MTB.

Table (2): Patients type distribution of the study group.

\begin{tabular}{|l|l|}
\hline Patients Type & Total $(\mathbf{N}=\mathbf{2 0 7})$ \\
\hline New & $193(93.2 \%)$ \\
\hline Contact & $7(3.4 \%)$ \\
\hline Relapse & $4(1.9 \%)$ \\
\hline Treatment fail & $3(1.5 \%)$ \\
\hline
\end{tabular}

This distribution may refer to a large group of patients with MTB complicated by pleural effusion.

Table (3): Specimen type distribution of the study group.

\begin{tabular}{|l|l|}
\hline Specimen type & $\begin{array}{l}\text { Total } \\
(\mathbf{N}=207)\end{array}$ \\
\hline $\begin{array}{l}\text { Broncho- alveolar lavage } \\
\text { (BAL) }\end{array}$ & $74(35.75 \%)$ \\
\hline Sputum (SP) & $51(24.64 \%)$ \\
\hline Pleural effusion (PE) & $82(39.61 \%)$ \\
\hline
\end{tabular}

This table shows that L.J. was more sensitive than AFB stain for detection of MTB 31 and 17 (15 and $8.2 \%$ ); respectively.

Table (4): LJ distribution of the study group.

\begin{tabular}{|l|l|}
\hline LJ & Total (N=207) \\
\hline Results & \\
Negative & $176(85.0 \%)$ \\
Positive & $31(15.0 \%)$ \\
\hline STR & $4 / 31(12.9 \%)$ \\
$R$ & $27 / 31(87.1 \%)$ \\
$S$ & \\
\hline INH & $5 / 31(16.1 \%)$ \\
$R$ & $26 / 31(83.9 \%)$ \\
$S$ & \\
\hline RIF & $3 / 31(9.7 \%)$ \\
$R$ & $28 / 31(90.3 \%)$ \\
$S$ & \\
\hline ETH & $31 / 31(100.0 \%)$ \\
$S$ & \multicolumn{2}{|l}{} \\
\hline
\end{tabular}

This table shows 34 (16.4\%) out of 207 tested samples were positive using MGIT system for $M$. tuberculosis growth detection. Four, five and three M. tuberculosis isolates were resistant to STR, INH and 
RIF; respectively. All the 34 isolates were sensitive to ETH.

Table (5): MGIT distribution of the study group.

\begin{tabular}{|l|l|}
\hline MGIT & Total $(\mathbf{N}=207)$ \\
\hline Results & \\
Negative & $173(83.6 \%)$ \\
Positive & $34(16.4 \%)$ \\
\hline STR & \\
$R$ & $4 / 34(11.8 \%)$ \\
$S$ & $30 / 34(88.2 \%)$ \\
\hline INH & \\
$R$ & $5 / 34(14.7 \%)$ \\
$S$ & $29 / 34(85.3 \%)$ \\
\hline RIF & \\
$R$ & $3 / 34(8.8 \%)$ \\
$S$ & $31 / 34(91.2 \%)$ \\
\hline ETH & \\
$S$ & $34 / 34(100.0 \%)$ \\
\hline
\end{tabular}

This table shows that Genexpert was less sensitive than MGIT and LJ for detection of MTB (12.6, 16.4 and $15 \%$ ) respectively. But was more sensitive for detection of RIF resistance than MGIT and $\mathrm{LJ}$ (11.5, 8.8 and 9.7\%); respectively.
Table (6): Genexpert (Gx) distribution of the study group.

\begin{tabular}{|l|l|}
\hline Gx & Total $(\mathrm{N}=207)$ \\
\hline Result & \\
Negative & $181(87.4 \%)$ \\
Positive & $26(12.6 \%)$ \\
\hline RIF & \\
R & $3 / 26(11.5 \%)$ \\
S & $23 / 26(88.5 \%)$ \\
\hline
\end{tabular}

This table shows no statistically significant difference between Gx, LJ and MGIT according to RIF resistance.

Table (7): Comparison between Gx, LJ and MGIT according to RIF resistance.

\begin{tabular}{|l|l|l|l|l|l|}
\hline RIF & $\begin{array}{l}\mathbf{G x} \\
(\mathbf{N}=\mathbf{2 6})\end{array}$ & $\begin{array}{l}\text { LJ } \\
(\mathbf{N}=31)\end{array}$ & $\begin{array}{l}\text { MGIT } \\
(\mathbf{N}=34)\end{array}$ & $\mathbf{x 2}$ & $\begin{array}{l}\mathbf{p}- \\
\text { value }\end{array}$ \\
\hline $\mathrm{R}$ & 3 & 3 & 3 & 0.124 & 0.938 \\
\hline $\mathrm{S}$ & 23 & 28 & 31 & & \\
\hline
\end{tabular}

This table shows highly statistically significant difference between Gx, LJ and MGIT according to duration of results (day).

Table (8): Comparison between Gx, LJ and MGIT according to duration of results (day).

\begin{tabular}{|l|l|l|l|l|l|}
\hline Result (day) & Gx $(\mathbf{N = 2 6})$ & $\begin{array}{l}\text { LJ } \\
(\mathbf{N}=\mathbf{3 1})\end{array}$ & MGIT $(\mathbf{N}=\mathbf{3 4})$ & ANOVA & p-value \\
\hline Mean \pm SD & $0.11 \pm 0.03$ & $41.10 \pm 11.61$ & $23.88 \pm 10.87$ & 11.128 & $<0.001$ \\
\hline
\end{tabular}

This table shows statistically significant correlation between specimen type and positive results AFB only.

Table (9): The correlation of specimen's type to positive results AFB, Gx, LJ and MGIT.

\begin{tabular}{|c|c|c|c|c|c|c|c|}
\hline \multirow{2}{*}{\multicolumn{2}{|c|}{ Positive Results }} & \multicolumn{3}{|c|}{ Specimen type } & \multirow{2}{*}{ Total } & \multicolumn{2}{|c|}{ Chi-square test } \\
\hline & & BAL & SP & PE & & $\mathbf{x} 2$ & p-value \\
\hline \multirow{2}{*}{ AFB } & No. & 7 & 8 & 2 & 17 & \multirow{2}{*}{7.558} & \multirow{2}{*}{$0.023(\mathrm{~S})$} \\
\hline & $\%$ & $41.18 \%$ & $47.06 \%$ & $11.76 \%$ & $100.0 \%$ & & \\
\hline \multirow{2}{*}{ Gx } & No. & 9 & 10 & 7 & 26 & \multirow{2}{*}{3.526} & \multirow{2}{*}{0.172 (NS) } \\
\hline & $\%$ & $34.62 \%$ & $38.46 \%$ & $26.92 \%$ & $100.0 \%$ & & \\
\hline \multirow{2}{*}{ L.J. } & No. & 12 & 11 & 8 & 31 & \multirow{2}{*}{3.586} & \multirow{2}{*}{0.167 (NS) } \\
\hline & $\%$ & $38.71 \%$ & $35.48 \%$ & $25.81 \%$ & $100.0 \%$ & & \\
\hline \multirow{2}{*}{ MGIT } & No. & 15 & 8 & 11 & 34 & \multirow{2}{*}{1.359} & \multirow{2}{*}{$0.507(\mathrm{NS})$} \\
\hline & $\%$ & $44.12 \%$ & $23.53 \%$ & $32.35 \%$ & $100.0 \%$ & & \\
\hline
\end{tabular}

- AFB: Sensitivity of 50\% specificity of $97.8 \%$ positive predictive value of $76.5 \%$, negative predictive value of $93.2 \%$ with diagnostic accuracy of $89.8 \%$.

- LJ: Sensitivity of $84.6 \%$ specificity of $95 \%$ positive predictive value of $71 \%$, negative predictive value of $97.7 \%$ with diagnostic accuracy of $93.7 \%$.

- MGIT: Sensitivity of $76.9 \%$ specificity of $92.3 \%$ positive predictive value of $58.8 \%$, negative predictive value of $96.5 \%$ with diagnostic accuracy of $90.3 \%$.

Table (10): Diagnostic Performance of AFB, LJ and MGIT in Discrimination of Gx. 
Ahmed Elsayed et al.

\begin{tabular}{|c|c|c|c|c|c|c|c|}
\hline \multirow[b]{2}{*}{ Results } & \multicolumn{2}{|c|}{ Gx Results } & \multirow[b]{2}{*}{ Sens. } & \multirow[b]{2}{*}{ Spec. } & \multirow[b]{2}{*}{ PPV } & \multirow[b]{2}{*}{ NPV } & \multirow[b]{2}{*}{ AUC } \\
\hline & $\begin{array}{l}\text { Positive } \\
(\mathrm{N}=26)\end{array}$ & $\begin{array}{l}\text { Negative } \\
(\mathrm{N}=\mathbf{1 8 1})\end{array}$ & & & & & \\
\hline $\begin{array}{l}\text { AFB } \\
\text { Positive } \\
\text { Negative }\end{array}$ & $\begin{array}{l}13 \\
13 \\
\end{array}$ & $\begin{array}{l}4 \\
177 \\
\end{array}$ & $50.0 \%$ & $97.8 \%$ & $76.5 \%$ & $93.2 \%$ & $89.8 \%$ \\
\hline $\begin{array}{l}\text { LJ } \\
\text { Positive } \\
\text { Negative }\end{array}$ & $\begin{array}{l}22 \\
4\end{array}$ & $\begin{array}{l}9 \\
172 \\
\end{array}$ & $84.6 \%$ & $95.0 \%$ & $71.0 \%$ & $97.7 \%$ & $93.7 \%$ \\
\hline $\begin{array}{l}\text { MGIT } \\
\text { Positive } \\
\text { Negative }\end{array}$ & $\begin{array}{l}20 \\
6\end{array}$ & $\begin{array}{l}14 \\
167\end{array}$ & $76.9 \%$ & $92.3 \%$ & $58.8 \%$ & $96.5 \%$ & $90.3 \%$ \\
\hline
\end{tabular}

- AFB: Sensitivity of $38.7 \%$, specificity of $97.2 \%$, positive predictive value of $70.6 \%$, negative predictive value of $90.0 \%$, with diagnostic accuracy of $88.4 \%$.

- GX: Sensitivity of $71.0 \%$, specificity of $97.7 \%$, positive predictive value of $84.6 \%$, negative predictive value of $95.0 \%$ with diagnostic accuracy of $93.7 \%$.

- MGIT: Sensitivity of $90.3 \%$, specificity of $96.6 \%$, positive predictive value of $82.4 \%$, negative predictive value of $98.3 \%$ with diagnostic accuracy of $95.7 \%$.

Table (11): Diagnostic Performance of AFB, Gx and MGIT in Discrimination of LJ.

\begin{tabular}{|c|c|c|c|c|c|c|c|}
\hline \multirow{2}{*}{ Results } & \multicolumn{2}{|c|}{ LJ Results } & \multirow{2}{*}{ Sens. } & \multirow{2}{*}{ Spec. } & \multirow{2}{*}{ PPV } & \multirow{2}{*}{ NPV } & \multirow{2}{*}{ AUC } \\
\hline & Positive & Negative & & & & & \\
\hline $\begin{array}{l}\text { AFB } \\
\text { Positive } \\
\text { Negative }\end{array}$ & $\begin{array}{l}12 \\
19 \\
\end{array}$ & $\begin{array}{l}5 \\
171 \\
\end{array}$ & $38.7 \%$ & $97.2 \%$ & $70.6 \%$ & $90.0 \%$ & $88.4 \%$ \\
\hline $\begin{array}{l}\mathbf{G x} \\
\text { Positive } \\
\text { Negative }\end{array}$ & $\begin{array}{l}22 \\
9\end{array}$ & $\begin{array}{l}4 \\
172\end{array}$ & $71.0 \%$ & $97.7 \%$ & $84.6 \%$ & $95.0 \%$ & $93.7 \%$ \\
\hline $\begin{array}{l}\text { MGIT } \\
\text { Positive } \\
\text { Negative }\end{array}$ & $\begin{array}{l}28 \\
3\end{array}$ & $\begin{array}{l}6 \\
170\end{array}$ & $90.3 \%$ & $96.6 \%$ & $82.4 \%$ & $98.3 \%$ & $95.7 \%$ \\
\hline
\end{tabular}

\section{DISCUSSION}

Tuberculosis (TB) remains one of the most fatal infectious diseases worldwide. The emergence of drug-resistant strains of Mycobacterium tuberculosis has put status of TB to threatening levels. Multidrugresistant TB (MDR-TB) is caused by M. tuberculosis complex (MTBC) strains that are resistant to at least two first-line anti-tuberculosis (anti-TB) drugs, isoniazid (INH) and rifampicin (RIF). The Global Extensively Drug-Resistant Tuberculosis (XDR-TB) Task Force of the World Health Organization (WHO) stated in 2006 that XDR-TB is a form of MDR-TB defined as resistant to at least any of the fluoroquinolones and at least one of the injectable anti-TB drugs (kanamycin, capreomycin, and amikacin) ${ }^{(10)}$.

Diagnosis of tuberculosis is a challenge, early diagnosis and prompt treatments of TB are crucial to reduce morbidity and mortality, secondary drug resistance, and transmission of TB. Despite low sensitivity in detection of Mycobacterium tuberculosis, acid-fast sputum smear remains the main diagnostic method in most countries, especially in resource limited settings. In HIV infected patients with pulmonary TB, 24-61\% have acid-fast negative sputum smear. Mycobacterial culture is the gold standard and the most sensitive method for TB diagnosis; however, the use in clinical practice is

limited due to a slow turnaround time, biosafety requirements, and high cost ${ }^{(\mathbf{1 1})}$.

In 2011, WHO endorsed the wide use of Xpert MTB/RIF assay, a fully automated diagnostic molecular test using real-time polymerase chain reaction (PCR) technology to simultaneously detect $M$. tuberculosis and rifampicin resistance mutations in the rpoB gene. This assay can provide the results within 2 hours. Several studies have demonstrated that Xpert assay is highly sensitive and specific in diagnosis of both pulmonary and extra pulmonary TB. Furthermore, Xpert assay was shown to be costeffective for TB diagnosis, compared to microscopy in low and middle income settings. Therefore, Xpert assay is strongly recommended as the initial diagnostic test in individuals suspected of having 
multidrug resistant (MDR) TB and in those with HIV/TB co-infection. It is also recommended as a follow-on test in TB-suspected patients with acid-fast negative sputum smear ${ }^{(\mathbf{1 2})}$.

In this study 207 Egyptian subjects were chosen without bias either by age or by gender only by suspected by a physician according to this study \{male $(56.5 \%)$ and female $(43.5 \%)\}$ of gender, with age 490 with mean age $36.72 \pm 20.79$, subdivided to 23 patients less than 10 years, 29 patients between 10-19, 28 patient between 20-29, 36 patients between 30- 39, 25 patients between 40-49, 31 patients between 50-59 and 35 patients 60 years or more.

The patients were divided into four groups, the new cases $(93.2 \%)$, contact to patients $(3.4 \%)$, relapse after complete the treatment $(1.9 \%)$ and Treatment failure $(1.5 \%)$. The specimens were broncho alveolar lavage (35.75\%), sputum (24.64\%) and pleural effusion (39.61\%).

In this study, it was found that MGIT culture is most sensitive then $\mathrm{LJ}$ culture then Genexpert and least sensitive is $\mathrm{ZN}$ stain.

These results were collectively as the following studies In Zhang et al., ${ }^{(13)}$.

This study revealed that there was a significant difference between mean of turnaround time of Gene Xpert, LJ and MGIT 0.11, 41.1 and 23.88 days; respectively.

This nearly agreed with Rakh and Elshahawy ${ }^{(14)}$ in which the turnaround time for Genexpert and MGIT were 0.1 and 23.5 days.

The result of these study revealed that the sensitivity of the different techniques for the diagnosis of tuberculosis depend on specimen's types whether sputum, bronchial lavage or pleural effusion.

These results agreed with Saeed et al., (15), Pandey et al., ${ }^{(16)}$, Albay et al., ${ }^{(17)}$ and Narute et al. (18).

This result revealed that AFB in comparison to Lowenstein-Jensen: Sensitivity $38.7 \%$, specificity of $97.2 \%$, positive predictive value $70.6 \%$, and negative predictive value $90.0 \%$, with diagnostic accuracy $88.4 \%$.

The result of AFB sensitivity nearly agreed with

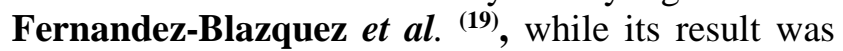
$41 \%$, and differ from Saeed et al. ${ }^{(15)}$, Rice et al. ${ }^{(20)}$ which result was $22.3,64.9$ and $22.2 \%$; respectively. The difference between last two studies and the present study may be because they did their stain directly and in this study it was done after concentration and decontamination.

The result of specificity of AFB nearly the same with Fernandez-Blazquez et al. ${ }^{(19)}$, Saeed et al. (15).

The result of this study not matched with the study of Rice et al. ${ }^{(20)}$ where the specificity of AFB was77.8\%; this may be happened due to this work happened in different countries and with different sample size.

The results for Lowenstein-Jensen in comparison to MGIT were sensitivity $82.4 \%$, specificity $98.3 \%$ positive predictive value $90.3 \%$, and negative predictive value $96.6 \%$ with diagnostic accuracy $95.7 \%$.

According to Jing $\boldsymbol{e t}$ al. ${ }^{(21)}$ sensitivity and specificity of $\mathrm{LJ}$ in comparison to MGIT were $67 \%$ and $90 \%$; respectively may be due to worked in other country.

The results for MGIT in comparison to LJ were Sensitivity of $90.3 \%$, specificity of $96.6 \%$, positive predictive value of $82.4 \%$, and negative predictive value of $98.3 \%$ with diagnostic accuracy of $95.7 \%$.

These results near the results of Rakh and Abdel Hakeem (22) which were $85.4 \%, 99.4 \%, 94.6 \%$ and $98.5 \%$; respectively,

It differs from result of Lin et al. ${ }^{(23)}$ of where $98.8 \%, 100 \%, 100 \%$, and $99.1 \%$; respectively. In Bangladesh by Sebastian et al. ${ }^{(24)}$ Taking LJ solid culture as reference gold standard the sensitivity of MGIT liquid culture was $75 \%$, specificity was $93 \%$, positive predictive value was $72 \%$ and negative predictive value was $94 \%$. The difference between them and present study may be due to they were done in other countries.

In this study the sensitivity, specificity, positive predictive value and negative predictive value of Genexpert in comparison to LJ were $71.0 \%, 97.7 \%$, $84.6 \%$, and $95.0 \%$; respectively.

The result of the sensitivity of Genexpert in this study, near the result of Fernandez et al. ${ }^{(18)}$, Shah et al. ${ }^{(25)}$, Agrawal et al. ${ }^{(26)}$ and Narute et al. ${ }^{(18)}$ which were 81, 78\%, 79.8, 77.3\%; respectively

And differ from the results of Rakh and Abdel Hakeem (21), Saeed et al. (14), Mafort et al. (27), Pachpute et al. ${ }^{(28)}$ and Pandey et al. (16) 97.6, 97.5, 93, $97 \%$ and $89 \%$; respectively.

The result of the specificity, positive predictive value and negative predictive value of Genexpert in comparison to LJ is near the result of Rakh and Abdel Hakeem ${ }^{(21)}$ where the results were $100 \%, 90.7 \%$ and 100\%; respectively, Agrawal et al. ${ }^{(26)}$ where results were $93.1 \%, 78.5 \%$ and $96 \%$; respectively. Pachpute et al. published in 2018 that specificity, positive predictive value and negative predictive value of Genexpert in comparison to LJ were $96 \%, 95 \%$ and 98\%; respectively. The result of Pandey et al. (16) specificity of $95 \%$, PPV of $89 \%$ and NPV of $95 \%$ using LJ culture as a reference standard.

In this study discovered that no statistically significant difference between Gx, LJ and MGIT according to RIF resistance.

This result nearly agreed with all the following Rice et al. ${ }^{(20)}$, Shetye et al. ${ }^{(29)}$, Lombardi et al. ${ }^{(30)}$, Shah 
et al. ${ }^{(25)}$ and Narute et al. ${ }^{(18)}$ which find 99.2, 97.8, 100,100 and $100 \%$.

\section{CONCLUSION}

Based upon the results of this study, it could be concluded that:

- Although the conventional methods remain the gold standard for diagnosing pulmonary $\mathrm{TB}$, delayed diagnostic times demand for more rapid and sensitive nucleic acid amplification techniques.

- Genexpert (MTB/RIF) assay is simple, rapid and accurate method for detecting mycobacterial tuberculosis.

- The assay is as sensitive as conventional drug sensitivity test for the diagnosis of MDR-TB and simultaneously detects MTBC and RIF's resistance. This assay is less dependent on the operator's skills, and staff with minimal training can use the Equipment.

- Although, Genexpert (MTB/RIF) assay has these advantages, similar to other tests for MTB, a negative result cannot exclude the diagnosis of TB.

- Also, patients with positive results can be assessed comprehensively with results of Ziehl-Neelsen smear test, culture, clinical symptoms and radiographic evidence.

\section{RECOMMENDATIONS}

It is recommended that:

- The extremely helpful Genexpert diagnostic tool should be implemented for screening and management of MDR-TB in TB endemic countries.

- Further work is needed for improving sensitivity, specificity and reproducibility of the Genexpert test and to make it more users friendly and cost effective.

\section{REFERENCES}

1. Brooks G, Carroll K, Stephen A and Timothy A (2010): Jawetz Melnick \& Adelbergs Medical Microbiology 25e - McGraw Hill Professional.

2. Van Embden J, Cave M, Crawford J et al. (1993): Strain identification of Mycobacterium tuberculosis by DNA fingerprinting: recommendations for a standardized methodology. J. Clin. Microbiol., 31: 406409.

3. Zadeh $C$ and Tayara $L$ (2018): Tuberculosis at Raffic Hariri University Hospital (RHUH) during 10 years period 2005-2015, cross sectional, observational study. Indian Journal of Tuberculosis, 65(3), 225-232.

4. World Health Organization (2017): Global tuberculosis report 2016. Guidelines for the treatment of drug-susceptible tuberculosis and patient care. http://www.who.int/tb/publications/global_report/en/
5. Steingart K. R, Ramsay A, Pai M (2007): Optimizing sputum smear microscopy for the diagnosis of pulmonary tuberculosis. Expert Rev. Anti Infect. Ther., 5:327-331

6. World Health Organization (2008): Interim policy guidance on drug susceptibility testing (DST) of second-line anti-tuberculosis drugs. World Health Organization, Geneva, Switzerland. http://www.WHO/HTM/TB/2008.392.

7. Piateka AS, Maarten V, Heather A, Coggind W, Manuela R, Sanne V, Shinnicke TM, Mukadia Y (2013): GeneXpert for TB diagnosis: planned and purposeful implementation" Glob Health Sci Pract., 1 (1): 18-23.

8. Egyptian National Tuberculosis Control Program (2015): Guideline for tuberculosis diagnosis and treatment.

http://www.ccs.gov.eg/ntp/publications_guidelines_ph ysicians_ntp.htm.

9. Genexpert manual (2013): Cepheid international HPM 40003

https://www.ghdonline.org/drtb/discussion/genexpertas-trusted-testing-tool.

10. Tekin K, Albay A, Simsek H, Sig AK, Guney M (2017): Evaluation of the BACTEC MGIT 960 SL DST Kit and the GenoType MTBDRsl Test for Detecting Extensively Drug-resistant Tuberculosis Cases. The Eurasian journal of medicine, 49 (3): 183.

11. van Kampen SC, Anthony RM, Klatser PR (2010): The realistic performance achievable with mycobacterial automated culture systems in high and low prevalence settings. BMC infectious diseases, 10(1): 94.

12. Pinyopornpanish K, Chaiwarith R, Pantip C, Keawvichit R, Wongworapat $K$, Sirisanthana $T$ (2015): Comparison of Xpert MTB/RIF assay and the conventional sputum microscopy in detecting Mycobacterium tuberculosis in Northern Thailand. Tuberculosis research and treatment .https://www.ncbi.nlm.nih.gov/pmc/articles/PMC4430 669.

13. Zhang Q, Zhang Q, Sun B, Liu C, Su A, Wang X, Hou G (2018): GeneXpert MTB/RIF for rapid diagnosis and rifampin resistance detection of endobronchial tuberculosis. Respirology, $1: 218-233$.

14. Rakha $E$ and Elshahawy $H$ (2018): Dramatic shortening of the diagnosis of multidrug-resistant tuberculosis by the detection of rifampicin resistance using a genotypic method: GeneXpert MTB/RIF assay. Comparative Clinical Pathology, 27: 1-6.

15. Saeed M, Iram S, Hussain S, Ahmed A, Akbar M, Aslam M (2017): GeneXpert: A new tool for the rapid detection of rifampicin resistance in mycobacterium tuberculosis. JPMA. The Journal of the Pakistan Medical Association, 67(2): 270-274.

16. Pandey S, Congdon J, McInnes B, Pop A, Coulter C (2017): Evaluation of the GeneXpert MTB/RIF assay on extrapulmonary and respiratory samples other than sputum: a low burden country experience. Pathology, 49(1): 70-74.

17. Albay A, Guney M, Tekin K, Kisa O Sig A (2016): Evaluation of the geneXpert MTB/RIF assay for early 
diagnosis of tuberculosis and detection of rifampicin resistance in pulmonary and extrapulmonary specimens. Cukurova Medical Journal, 41(3): 548-553.

18. Narute S, Salgia $K$, Singhal $P$, Kalley V (2015): Comparative study of gene Xpert MTB/RIF, smear microscopy and TB MGIT culture in diagnosis of tuberculosis in India, http://www.erj.ersjournals.com/content/46/suppl_59/P A1533

19. Fernandez-Blazquez A, Sabater C, GarciaBernardo A, Garcia-Garcia JM, Villa RL, Pando M Palacios JJ (2018): Contribution of Xpert MTB/RIF to the Diagnosis of Tuberculosis in a Low-Prevalence Country Over a Period of 7 Years (2010-2017). In A25. TUBERCULOSIS MANAGEMENT: NEW INSIGHTS (pp. A1141-A1141). American Thoracic Society,

www.atsjournals.org/doi/abs/10.1164/ajrccmconferenc e.2018.197.1_MeetingAbstracts.A1141

20. Rice JP, Seifert M, Moser KS, Rodwell TC (2017): Performance of the Xpert MTB/RIF assay for the diagnosis of pulmonary tuberculosis and rifampin resistance in a low-incidence, high-resource setting. PloS one, 12(10): e0186139

21. Jing H, Lu Z, Deng Y, Gao D, Li L, Graviss EA, Ma X (2017): Evaluation of Xpert MTB/RIF in detection of pulmonary and extrapulmonary tuberculosis cases in China. Int J Clin Exp Pathol, 10 (4): 4847-4851.

22. Rakha EB and Abdel Hakeem MA (2017): GeneXpert MTB/RIF Assay: A Revolutionizing Method for Rapid Molecular Detection of Mycobacterium Tuberculosis in Comparison to Other Conventional Methods. Int. J. Curr. Microbiol. App. Sci., 6(10): 2573-2580.

23. Lin SY, Hwang SC, Yang YC, Wang CF, Chen YH, Chen TC, Lu PL (2016): Early detection of Mycobacterium tuberculosis complex in BACTEC MGIT cultures using nucleic acid amplification. European Journal of Clinical Microbiology \& Infectious Diseases, 35(6): 977-984.
24. Sebastian G, Tripathy SK, Vishwanatha T, Kumar P (2016): Recovery rate of mycobacterium tuberculosis by BACTEC MGIT 960 liquid culture system from sputum samples of presumptive pulmonary tuberculosis patients. World Journal of Pharmacy And Pharmaceutical Sciences, 5(8): 1646-1653.

25. Shah R, Uppe A, Nair G, Sharma A, Patel S. and Ravindran J (2017): Comparison studies of GENEXPERT versus MGIT culture of Bronchial Lavage in sputum smear negative suspected Pulmonary Tuberculosis. European Respiratory Journal, 50: PA2743.

26. Agrawal M, Bajaj A, Bhatia V, Dutt S (2016): Comparative study of GeneXpert with $\mathrm{ZN}$ stain and culture in samples of suspected pulmonary tuberculosis. Journal of clinical and diagnostic research: JCDR., 10(5): DC09.

27. Mafort TT, Rodrigues LS, Santos AP, Reis LV, Faria LF, Brito GM and Rufino $R$ (2017): Bronchoalveolar Lavage GeneXpert MTB/Rif® Performance In Smear-Negative Pulmonary Tuberculosis-A Tertiary Care Experience In Rio De Janeiro, Brazil. https://www.atsjournals.org/doi/pdf/10.1164/ajrccmco nference.2017.195.1_MeetingAbstracts.A2085

28. Pachpute $S$, Pachpute $P$ and Urhekar AD (2018): to study the diagnostic accuracy of genexpert mtb/rif assay for the diagnosis of pulmonary tuberculosis in a tertiary care hospital in navi Mumbai. international journal of scientific research, 12;7(2): 59-61.

29. Shetye S, Chheda P, Lad A and Matkar S (2017): performance of XPERT MTB/RIF ASSAY for detection of $\mathrm{m}$. tb in pulmonary and extra-pulmonary samples in Indian patients. SAARC Journal of Tuberculosis, Lung Diseases and HIV/AIDS., 14(1): 713.

30. Lombardi G, Di Gregori V, Girometti N, Tadolini M, Bisognin F, Dal Monte P (2017): Diagnosis of smear-negative tuberculosis is greatly improved by Xpert MTB/RIF, PloS one, 12(4): e0176186. 